\title{
Characterization of Enterococcus species in a tertiary care hospital
}

\author{
Nautiyal S, Jauhari S, Joshi G, Kataria V.K. \\ 1. Department of Microbiology, Shri Guru ram Rai Institute of Medical \& Health Sciences, India \\ 2. Department of Microbiology, Shri Guru ram Rai Institute of Medical \& Health Sciences, India \\ 3. Department of Microbiology, Shri Guru ram Rai Institute of Medical \& Health Sciences, India \\ 4. Department of Microbiology, Shri Guru ram Rai Institute of Medical \& Health Sciences,India
}

\begin{abstract}
Background \& objectives: Enterococci are a part of normal intestinal flora of humans and animals but are increasingly being recognized as important human pathogens. Although they cause only a few clinical infections in human beings since the beginning of antibiotic era, they have posed major therapeutic challenges to humans. Material \& methods: Various samples coming to Microbiology division of central laboratory such as blood, pus, urine etc. were included in the study. All the essential biochemical reactions needed to establish the identity of Enterococci spp. were put followed by antibiotic sensitivity testing by Kirby Bauer disc diffusion technique. Results: In our study we found the predominance of Enterococcus faecalis followed by E. faecium and E.columbae.44.4\% isolates were recovered from surgery ward predominantly from urine samples. Discussion: The present study was undertaken for phenotypic characterization of Enterococcus spp. Majority of cases belonged to surgery probably due to catheterization, surgical procedures and prolonged hospitalization.
\end{abstract}

Keywords: Enterococci, VRE, Hospital infection control,

\section{INTRODUCTION}

Enterococci, formerly classified with Streptococci, have been recognized to be of fecal origin since the beginning of this century. The genus Enterococcus comprises Gram positive cocci that are catalase negative, usually facultative anaerobic bacteria that grow in $6.5 \% \mathrm{NaCl}, 40 \%$ bile salts, $0.1 \%$ methylene blue milk and at $\mathrm{pH}$ 9.6. They grow at $10^{\circ} \mathrm{C}$ and $45^{\circ} \mathrm{C}$ and can resist 30 min at $60^{\circ} \mathrm{C} .(1,2,3)$

Over the past two decades, Enterococci have been identified as the agents of nosocomial infection with increasing frequency. $(4,5)$ These organisms have survived in the hospital environment due to their intrinsic resistance to several commonly used antibiotics and more importantly their ability to acquire resistance to all currently available antibiotics, either by mutation or by receipt of foreign genetic material through the transfer of plasmids and transposons $(6,7,8)$.

Vancomycin has been used as the drug of choice in many resistant strains of Gram positive bacterial infections, especially those caused by Enterococci. There has been an increase in number of Vancomycin Resistant Enterococci [VRE] in recent times. The organism can also horizontally transfer this resistant determinant to other Vancomycin-susceptible species $(9,10)$. Vancomycin inhibits Enterococci by binding to the D-alanine-D-alanine terminus of the cell wall precursors, compromising the synthesis of the bacterial cell wall. If the amino-acid composition of such terminus is altered, Vancomycin binds to it with much lower affinity. Resistance to Vancomycin is encoded by different clusters of genes referred to as the Vancomycin resistance gene clusters (e.g. VanA, VanB and VanC). Both high and low level Vancomycin resistance can occur in Enterococci. Low level Vancomycin resistance (VanC) is limited to 2 relatively non-virulent species: $E$. gallinarum and E. casseliflavus. High-level resistance (encoded by VanA and VanB genes) is related to $E$. faecalis and E. faecium. They are more problematic as it is frequently associated with resistance to multiple classes of antibiotics. The fourth Vancomycin resistance genotype, VanD described in strain of E. faecium exhibit moderate level of resistant to Vancomycin and Teicoplanin. The major phenotypes (referred to as VanA and $\operatorname{Van} B$ ) can be differentiated by the level of Vancomycin resistance and susceptibility to Teicoplanin (8). Resistance to Linezolid is slowly developing, posing several questions on the virulence factors and their survival mechanisms (11).

The Center for Disease Control and Prevention, in a survey on nosocomial infections, indicated that Enterococcus accounted for $13.9 \%$ infections, being next to Escherichia coli as a causative agent of hospital acquired urinary tract infections (12). Therefore, the same importance is given to the multidrug resistant Enterococcus species, like that of Methicillin Resistant Staphylococcus Aureus (MRSA) and Extended Spectrum Beta Lactamase (ESBL) producers, as nosocomial pathogens (13). 
Based on the above facts this study was conducted with the objectives to find out the predominant species of Enterococcus in our hospital settings, to find out the extent of Multi-drug resistance pattern of the isolates and to create a baseline to keep a check on emergence of VRE for future studies and for hospital infection control purpose.

\section{MATERIALS \& METHODS}

This study was carried out in the Department of Microbiology, of Shri Guru Ram Rai Institute of Medical and Health Sciences (SGRRIM\&HS) and Shri Mahant Indiresh Hospital (SMIH), Patel Nagar, Dehradun, from August 2014 to March 2015 over a period of 8 months.

A total of 45 isolates of Enterococci cultivated and identified from various samples like - Blood, Pus, Tip, Urine, Throat swab and HVS in the Microbiology division of Central Laboratory, at SMIH were included in this study.

After preliminary Gram staining of specimen, enrichment of samples was done in Brain Heart Infusion broth (BHI) and sub-culture was done on Blood agar \& McConkey agar. Catalase test and biochemical tests like bile esculin hydrolysis, salt tolerance test using $6.5 \% \mathrm{NaCl}$, PYR test (using $0.01 \%$ pdimethylaminocinnamaldehyde), Arginine Decarboxylation, sugar fermentation using D (-)Arabinose, DMannitol, L(-)Sorbose, D- Sorbitol and D (+) - Raffinose were carried out on colonies grown.

The antibiotic sensitivity was put as per CLSI guidelines using Kirby Bauer disc diffusion technique.(11)

\section{RESULTS}

It was observed that enterococcal species were predominantly isolated from 21-50 years of age group $24 / 45(53.33 \%)$, followed by the patients in the age group of $0-10 \mathrm{yrs}$ and 51-60yrs i.e $5 / 45(11.10 \%)$ each. While lower number of Enterococcus was isolated from age group 60yrs and above 7/45(15.55\%). More males 25/45 (55.50\%) than females 20/45 (44.50\%) were infected by Enterococcus species.

It was found that $44.40 \%$ of isolates were recovered from Surgery ward followed by patients from Medicine and Gynae \& Obstretics $15.60 \%$ each, while fewer isolates were recovered from Pediatric ward, Orthopedics ward and OPD as well.

Table 1 shows that Enterococcus were isolated predominantly from urine (46.70\%), followed by pus and blood with $(28.29 \%) \&(7 \%)$. Table 2 shows the species identification of Enterococcus isolates with predominance of E.faecalis $(75.60 \%)$.

\section{DISCUSSION}

Enterococcus is one of the most common cause of the nosocomial infection probably due to the inherent resistance to antibiotics (Cephalosporins) and with increasing frequency of antimicrobial resistance to most of the currently used antibiotics, ability to adhere to indwelling medical devices and ability to survive adverse environmental conditions (14).

In today's era, correct speciation is very important since there is variation in resistance to antibiotics expressed by various enterococcal species. Therefore the present study was undertaken for phenotypic characterization of Enterococcus.

Ward wise distribution of the isolates studied show that $42 / 45$ (93.33\%) were admitted to various wards while only $3 / 45(6.67 \%)$ cases were outdoor patients. In the wards, majority of the cases belonged to surgery 20/45 (44.40\%), probably due to catheterization, surgical procedures and longer hospitalizations. Similar findings have been observed by other workers also ranging from $72-80 \%$ in indoor cases $(15,16,17)$.

Of the various specimens from which Enterococci were isolated, it was observed that 21/45(46.70\%) were from urine. Isolation of Enterococci predominantly from urine has also been reported from various studies also.(15, 18) The most probable reason for high isolation rate of Enterococci from UTI cases could be due to the close proximity of anal opening to urethra as Enterococci reside as commensals in GIT. Urinary catheterization as required in some cases may also have contributed to higher isolation of Enterococci from urine specimens.

The predominant species of Enterococci isolated in the present study was Enterococcus faecalis 34/45 (75.60\%) followed by Enterococcus.faecium 5/45 (11.10\%). Similar observations were reported by various workers $(14,15,18)$. In the present study E.columbae, E.durans, E.canis and E.sulfureus were also isolated. Udo EE et al and Chaudhary U et al (18) also have described these species as pathogenic though of less potential.

The present study showed that 26/45(57.7\%) of Enterococcal isolates were resistant to ampicillin and 25/45(55.5\%) to amoxicillin-clavulanic acid, 35/45(77.7\%) isolates were resistant to gentamicin and doxycycline. (19) This type of resistance pattern with gentamicin was also reported by Nepal et al (14). 3/45 (6.66\%) Vancomycin Resistant Enterococci have been isolated, two from blood and one from pus.

In the present study we have phenotypically isolated one strain of Van A, and two strains of Van B. Possibility of emergence of VRE in the present study could be due to the fact that majority of the isolates were 
from various wards. This raises the chance of the hospital resident flora infecting the patients. Since the hospital resident flora is constantly being exposed to a large group of antibiotics, therefore VRE of $6.66 \%$ has been reported in this study.

Tables

Table 1: Specimen-wise distribution of Enterococcal isolates studied $(n=45)$

\begin{tabular}{|l|c|}
\hline Specimen & Number(\%) \\
\hline Blood & $7(15.60)$ \\
\hline Pus & $13(28.29)$ \\
\hline Urine & $21(46.70)$ \\
\hline HVS & $2(4.50)$ \\
\hline Suction tip & $1(2.20)$ \\
\hline Throat swab & $1(2.20)$ \\
\hline Total & $\mathbf{4 5 ( 1 0 0 )}$ \\
\hline
\end{tabular}

Figures in parentheses denote percentage.

Table 5: Distribution of Enterococcal species isolated in the study $(n=45)$

\begin{tabular}{|l|c|}
\hline Species identified & Number (\%) \\
\hline E. faecalis & $34(75.60)$ \\
\hline E. faecium & $5(11.10)$ \\
\hline E.columbae & $3(6.70)$ \\
\hline E. durans & $1(2.20)$ \\
\hline E. canis & $1(2.20)$ \\
\hline E.sulfureus & $1(2.20)$ \\
\hline Total & $\mathbf{4 5 ( 1 0 0 )}$ \\
\hline
\end{tabular}

Figures in parentheses denote percentage.

\section{CONCLUSION}

Enterococcus thought to be a commensal in the past has gained importance in today's times. It has become an established pathogen causing nosocomial infection in a vast spectrum of illnesses. This study reveals a high isolation rate of Enterococci from indoor cases $(93.33 \%)$.

Of all the isolates studied it was found that $6.66 \%$ were Vancomycin resistant Enterococci (VRE). The emergence of VRE is a cause for great concern because of association of these infections with high mortality, the limited therapeutic options available for such infections and due to their potential to transfer Vancomycinresistance genes to other organisms. These infections then require newer antibiotics such as Linezolid and Tigecycline, which are not free of various side effects. VRE bacteremia thus prolongs the duration of hospital stay.

Therefore there is a need to upgrade our vigil against the emergence and upsurge of the previously known commensals turning into pathogens and also to prevent the development of high level resistance. Basic practices of barrier nursing and hand washing may go a long way in curtailing the spread of the multidrug resistant strains in hospital settings.

\section{REFERENCES}

[1]. Murray PR, Baron EJ, Jorgensen JH, Pfaller MA and Yolken RH. Manual of Clinical Microbiology; $8^{\text {th }}$ edt: $\operatorname{vol}(1)$.

[2]. Schleifer KH and Kilpper-Balz R.Transfer of Streptococcus faecalis and Streptococcus faecium to the Genus Enterococcus norn. rev. as Enterococcus faecalis comb. nov. and Enterococcus faecium comb. nov. Int. J. Syst. Bacteriol.1984; 34(1): 31-34.

[3]. Schleifer KH and Kilpper-Balz R. Molecular and chemotaxonomic approaches to the classification of Streptococci, Enterococci and Lactococci: A Review. System. Appl. Microbiol. 1987; 10(1): 1-19.

[4]. Mundy LM, Sahm DF and Gilmore M. Relationships between Enterococcal virulence and antimicrobial resistance. Clin. Microbiol. Rev. 2000; 13(4): 513-522. 
[5]. Clewell DB. Movable genetic elements and antibiotic resistance in Enterococci. Eur. J. Clin. Microbiol. Infect Dis. 1990; 9(2): 90-102.

[6]. Upadhyaya PMG, Ravikumar KL and Umapathy BL. Review of virulence factors of Enterococcus: an emerging nosocomial pathogen. Indian Journal of Medical Microbiology(2009); 27(4): 301-305.

[7]. Chlebicki MP and Kurup A. Vancomycin-resistant Enterococcus - A Review from a Singapore perspective. Ann. Acad. Med. Singapore. 2008; 37(10): 861-869.

[8]. Kainer MA, Devasia RA, Jones TF, Simmons BP, Melton K, et al. Response to Emerging Infection Leading to Outbreak of Linezolid Resistant Enterococci. Emerging Infectious Diseases; 2007: 13(7)

[9]. Desai PJ, Pandit D, Mathur M, Gogate A. Prevalence, identification and distribution of various species of Enterococci isolated from clinical specimens with special reference to urinary tract infection in catheterized patients. Indian J Med Microbiol. 2001; 19(3): 132- 137.

[10]. Sreeja S, Sreenivasa Babu PR, Prathab AG. The prevalence and the characterization of the Enterococcus species from various clinical samples in a tertiary care hospital. Journal of clinical and Diagnostic Research. 2012; 6(9): 1486-1488.

[11]. Koneman. Koneman's color atlas and textbook of diagnostic microbiology. $6^{\text {th }}$ edt.Philadelphia: Lippincott Williams and Wilkins: 2006.

[12]. Shabnam Qamer, Jonathan Sandoe, Kevin G Kerr. Use of colony morphology to distinguish different Enterococcal strains and species in mixed culture from clinical specimens. Journ. of Clin Micro. June 2003: p 2644-46.

[13]. Acharya A, Khanal B, Kanugo R and Mohpatra T. Characterisation and susceptibility patterns of clinically important Enterococcus species in Eastern Nepal, Nepal Med Coll J. 2007 Dec; 9(4): 250-254.

[14]. Nepal HP, Khanal B and Acharya A. Characterization and antimicrobial resistance of clinical isolates of Enterococci in tertiary care hospital in Eastern Nepal. Health Renaissance 2013; 11(1): 17-22.

[15]. Ujjwala B, Vilas T and Neena N. Identification of Enterococcus species isolated from clinical specimens and detection of Vancomycin resistant Enterococci. Int J Pharm Bio Sci 2014 April; 5(2): (B) 847-852.

[16]. Bose S, Ghosh KA and Barapatre R. Prevalence of drug resistance among Enterococcus species isolated from a tertiary care hospital. International Journal of medical and health sciences. 2012; 1(3): 38-44.

[17]. Palanisamy S, Karunakaran S and Narayanan Shankara. Antimicrobial resistance profile and characterisation of Enterococcus species from various clinical samples in a tertiary care hospital. Int $\mathbf{J}$ Med Res Health Sci. 2013; 2(3): 328-333.

[18]. Udo EE, Noura A, Phillips OA and Chugh TD. Species prevalence and antimicrobial resistance of Enterococci isolated in Kuwait hospitals. J Med Micobiol. 2003; 52: 163-168.

[19]. Suresh K, Saipriya B and Viswanath G. Isolation, Speciation and Determination of High Level Aminoglycoside Resistance of Enterococci among hospitalized patients in Davangere. National Journal of Laboratory Medicine. 2013 April; 2(1): 12-15. 